\title{
Stool Donor Body Mass Index Does Not Affect Recipient Weight After a Single Fecal Microbiota Transplantation for $C$. difficile Infection
}

\section{Short title: Donor BMI does not affect recipients' post-FMT weight}

Monika Fischer MD, $\mathrm{MSc}^{1}$, Dina Kao $\mathrm{MD}^{2}$, Zain Kassam MD, $\mathrm{MPH}^{3}$, Justin Smith $\mathrm{BSc}^{2}$, Thomas Louie $M D^{4}$, Brian Sipe $M D^{5}$, Michelle Torbeck $\mathrm{MD}^{1}$, Huiping $\mathrm{Xu} \mathrm{PhD}^{6}$, Fangqian Ouyang $\mathrm{MS}^{6}$ Dariush Mozaffarian MD, $\mathrm{DrPH}^{7}$, , and Jessica R. Allegretti MD, $\mathrm{MPH}^{8,9}$

1. Department of Gastroenterology, Indiana University School of Medicine, Indianapolis, IN

2. Division of Gastroenterology, University of Alberta, Edmonton, $A B$

3. OpenBiome, Somerville, MA

4. Division of Infectious Diseases, University of Calgary, Calgary, AB

5. Down25, Indianapolis, IN

6. Department of Biostatistics, The Richard M. Fairbanks School of Public Health and School of Medicine, Indiana University, IN

7. Tufts Friedman School of Nutrition Science \& Policy, Boston, MA

8. Brigham and Women's Hospital, Boston, MA

9. Harvard Medical School, Boston, MA

\section{Corresponding author:}

Monika Fischer, MD, MSc.

Division of Gastroenterology and Hepatology, Indiana University School of Medicine 550 N. University Blvd, Suite 1602, Indianapolis, IN 46202, USA.

E-mail: mofische@iu.edu

Phone: 317-318-5240

Fax: 319-944-0975

Funding: The study was funded in part by Indiana University Gastroenterology Division endowment to Monika Fischer

\section{Specific Author contribution:}

- Monika Fischer: study concept and design; acquisition of data; analysis and interpretation of data; drafting of the manuscript; critical revision of the manuscript for important intellectual content

- Brian Sipe: study concept and design; acquisition of data; critical revision of the manuscript for important intellectual content

- Michelle Torbeck: study concept and design; acquisition of data

This is the author's manuscript of the article published in final edited form as:

Fischer, M., Kao, D., Kassam, Z., Smith, J., Louie, T., Sipe, B., ... Allegretti, J. R. (2017). Stool Donor Body Mass Index Does Not Affect Recipient Weight After a Single Fecal Microbiota Transplantation for C. difficile Infection. Clinical 
- Huiping Xu and Fangqian Ouyang: analysis and interpretation of data; drafting of the method sections; critical revision of the manuscript for important intellectual content

- Dariush Mozaffarian: analysis and interpretation of data; critical revision of the manuscript for important intellectual content

- Zain Kassam: analysis and interpretation of data; drafting of the manuscript; critical revision of the manuscript for important intellectual content

- Jessica R. Allegretti: acquisition of data; analysis and interpretation of data; drafting of the manuscript; critical revision of the manuscript for important intellectual content

- Dina Kao: acquisition of data; analysis and interpretation of data; drafting of the manuscript; critical revision of the manuscript for important intellectual content

- Justin Smith: acquisition of data; analysis and interpretation of data; critical revision of the manuscript for important intellectual content

- Thomas Louie: acquisition of data; critical revision of the manuscript for important intellectual content

\section{COI:}

Jessica Allegretti is a scientific advisor and receives research support from Finch Therapeutics. Monika Fischer is a research consultant for Finch Therapeutics.

Zain Kassam is employed by OpenBiome and a research consultant and shareholder at Finch Therapeutics.

Justin Smith has no financial or personal COI relevant to the manuscript. Thomas Louie has no financial or personal COI relevant to the manuscript. Brian Sipe has no financial or personal COI relevant to the manuscript. Michelle Torbeck has no financial or personal COI relevant to the manuscript. Huiping Xu has no financial or personal COI relevant to the manuscript. Fangqian Ouyang has no financial or personal COI relevant to the manuscript. Dariush Mozaffarian has no financial or personal COI relevant to the manuscript. 


\section{Background}

Emerging data suggests obesity may be transmissible by gut microbiota. ${ }^{1} \mathrm{~A}$ study of germ-free mice reports an increase in adiposity by transferring human stool from twins discordant for obesity. ${ }^{2}$ Additionally, there has been a case report of a 34-pound weight gain following fecal microbiota transplantation (FMT) from a patient's overweight daughter. ${ }^{3}$ Given this data, current best practices exclude obese stool donors. However, prior to these recommendations, overweight and obese patient-selected donors (e.g. spouse) were used, and there is a paucity of data on the clinical impact. Accordingly, we assessed the change in body mass index (BMI) of FMT recipients successfully treated for recurrent Clostridium difficile infection (CDI) ( $\geq 3$ episodes) who received stool from obese, overweight and normal weight donors.

\section{Methods}

Data was aggregated from a randomized clinical trial (RCT) and an observational study assessing FMT in CDI. The RCT participants were randomized to receive FMT via capsule or colonoscopy. Medically documented BMI was obtained prior to CDI ( $\geq 6$ months before FMT), at FMT baseline, and post-FMT up to 48 weeks.

For the observational cohort, consecutive patients who underwent a single FMT by colonoscopy were included if they had a medically documented BMI prior to CDI ( $\geq 6$ months before FMT), at FMT baseline, and post-FMT up to 52 weeks. For both groups, patients without BMI data were excluded from the study.

In the observational cohort study, patients received stool from normal (BMI=18-24.9 kg/m²), overweight $\left(\mathrm{BMI}=25-29.9 \mathrm{~kg} / \mathrm{m}^{2}\right)$ or obese $\left(\mathrm{BMI} \geq 30 \mathrm{~kg} / \mathrm{m}^{2}\right)$ donors. In the $\mathrm{RCT}$, patients received stool from normal or overweight donors. Patient characteristics were compared using Pearson's chi-square test for categorical variables and ANOVA F-test for continuous variables. The change 
in patient BMI pre- and post-FMT was examined using a repeated-measures mixed effects model. Stool microbial composition on a subset of patients $(n=24)$ and donors (normal weight $[n=4]$, overweight $[n=2]$ ) were analyzed. PERMANOVA analysis was performed to compare the normal and overweight donor recipient groups, using Bray-Curtis distances and 999 permutations.

\section{Results}

Overall, this analysis included a total of 173 patients receiving successful FMT for CDI. The 103 RCT participants (mean age $57.4 \pm 18,70 \%$ women, $3 \%$ severe CDI) received stool from 7 volunteer donors, where 66 (64\%) participants received normal weight donor material and 37 (36\%) participants received overweight donor material. The time trends in BMI changes postFMT were not different between the normal and overweight donor groups $(p=0.88)$.

The 70 observational cohort participants (mean age $53.7 \pm 18,79 \%$ women, $11 \%$ severe CDI) received stool from 39 donors ( 5 volunteer and 34 patient-selected). Among the cohort, 25 patients (36\%) received normal weight donor, 30 (43\%) overweight donor and $15(21 \%)$ obese donor material. The time trend of BMI changes post-FMT were not different between the three groups: normal vs. overweight (Mean diff in weekly change: 0.002; 95\% $\mathrm{Cl}:-0.052,0.056$; $\mathrm{p}=0.94$ ), normal vs. obese (Mean diff in weekly change: $0.04 ; 95 \% \mathrm{Cl}:-0.03,0.1 ; p=0.25$ ), or overweight vs. obese (Mean diff in weekly change: $0.04 ; 95 \% \mathrm{Cl}:-0.03,0.09 ; \mathrm{p}=0.27$ ).

Overall, all patients experienced weight loss during CDI (pre-CDI BMI vs. FMT baseline) and increased BMI post-FMT, but did not exceed pre-CDI BMI (Figure 1a/b). Adjusted analysis controlling for patient characteristics including age, sex, CDI severity at the time of FMT, and 
stool donor type (patient-selected vs volunteer) yielded similar results as the unadjusted model. In addition, sensitivity analysis examining the clustering effect of patients who received stool from the same donor showed similar results. PERMANOVA analysis comparing the normal and overweight donor recipients did not find a statistically significant difference in microbial engraftment $(p=0.068)$ (Data not shown).

\section{Discussion}

Emerging evidence has linked the microbiome to obesity ${ }^{1}$ and metabolic syndrome. ${ }^{4,5}$ Accordingly, stool banks have adopted rigorous standards for donor screening including assessing obesity ${ }^{6}$; however, overweight and obese donors were previously used among early adopters. Analyzing aggregated data from an observational cohort and a RCT, we found no statistically significant differences in changes in recipient BMI based on donor BMI up to 48 weeks following a single FMT. Most importantly, although weight gain was seen following FMT, patients appear to have regained weight lost during their diarrheal illness, and not beyond their pre-CDI BMI irrespective of stool donor BMI. These results should be interpreted with some caution due to limited microbiome data, association with only a single rather than multiple FMTs, different FMT delivery modality, and aggregation of two similar but distinct study populations. To our knowledge, this is the first study to demonstrate that donor BMI does not impact FMT recipient BMI following a single FMT; however, further prospective research is required to confirm this phenomenon, and obese/overweight donors should continue to be excluded. 


\section{References}

1. Turnbaugh PJ, Ley RE, Mahowald MA, et al. An obesity-associated gut microbiome with increased capacity for energy harvest. Nature 2006;444:1027-1031.

2. Ridaura VK, Faith JJ, Rey FE, et al. Gut microbiota from twins discordant for obesity modulate metabolism in mice. Science (New York, N.Y.) 2013;341:1241214.

3. Alang N, Kelly CR. Weight gain after fecal microbiota transplantation. Open forum infectious diseases 2015;2:ofv004.

4. Vrieze A, Van Nood E, Holleman F, et al. Transfer of intestinal microbiota from lean donors increases insulin sensitivity in individuals with metabolic syndrome. Gastroenterology 2012;143:913-6.e7.

5. Kelly CR, Kahn S, Kashyap P, et al. Update on Fecal Microbiota Transplantation 2015: Indications, Methodologies, Mechanisms, and Outlook. Gastroenterology 2015;149:223-237.

6. Smith M, Kassam Z, Burgess J, et al. The International Public Stool Bank: A Scalable Model for Standardized Screening and Processing of Donor Stool for Fecal Microbiota Transplantation. Gastroenterology 2015;148 (4):S-211.

Figure 1. Estimated mean BMI (95\% confidence interval) based on the repeated measures mixed effects model 
(a). RCT Data

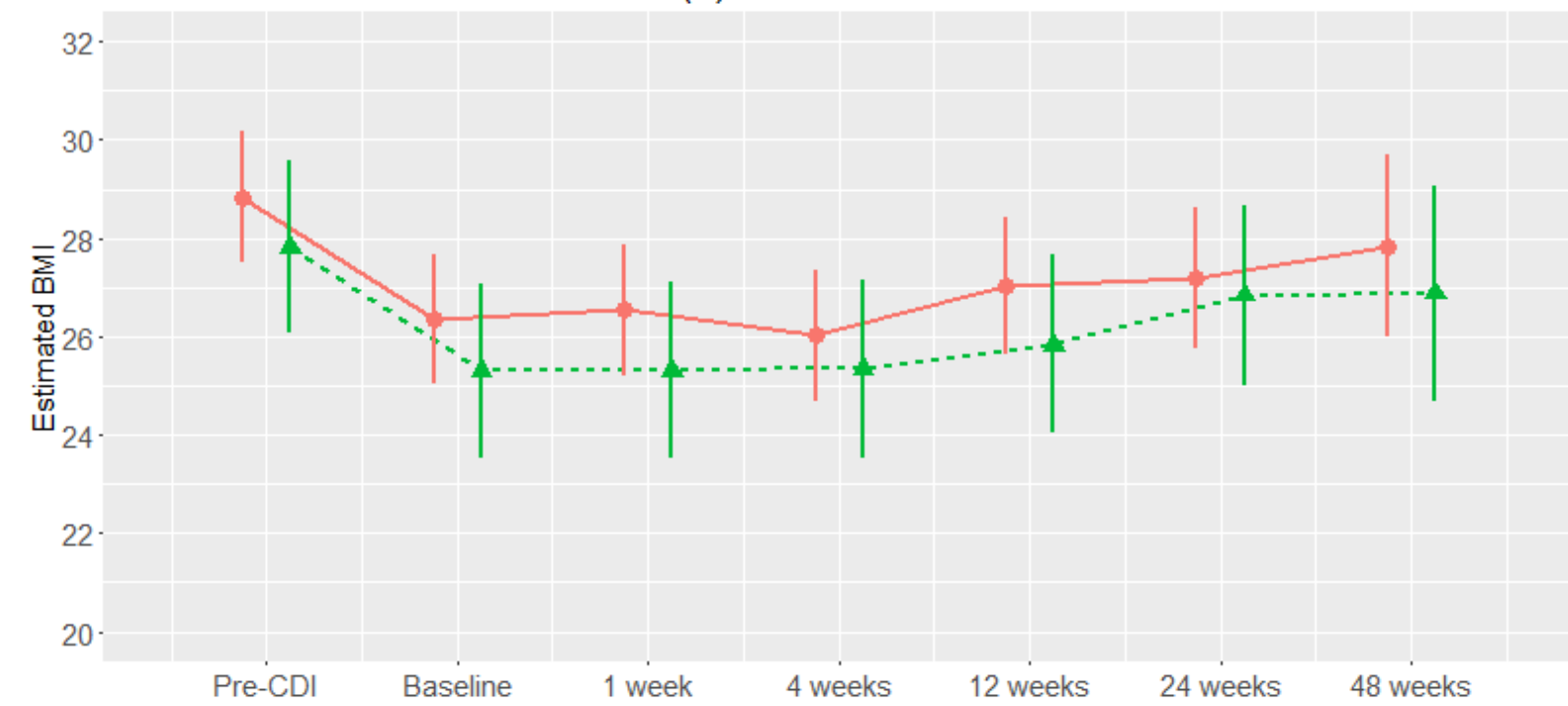




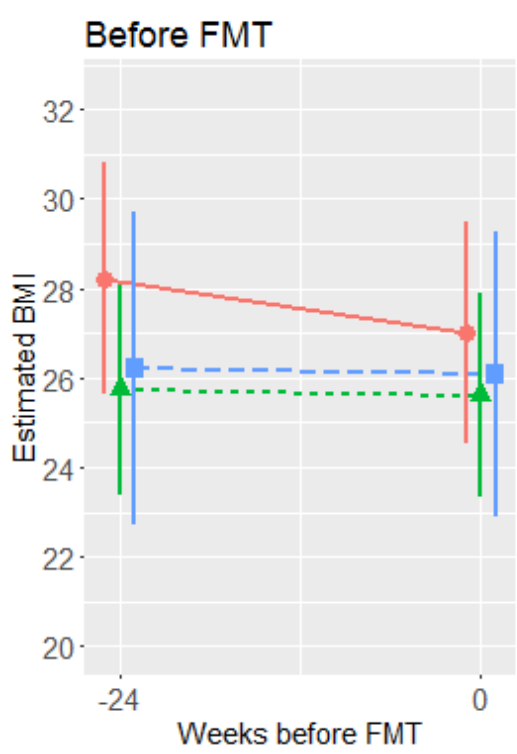

\section{(b). Observational Cohort}

\section{After FMT}

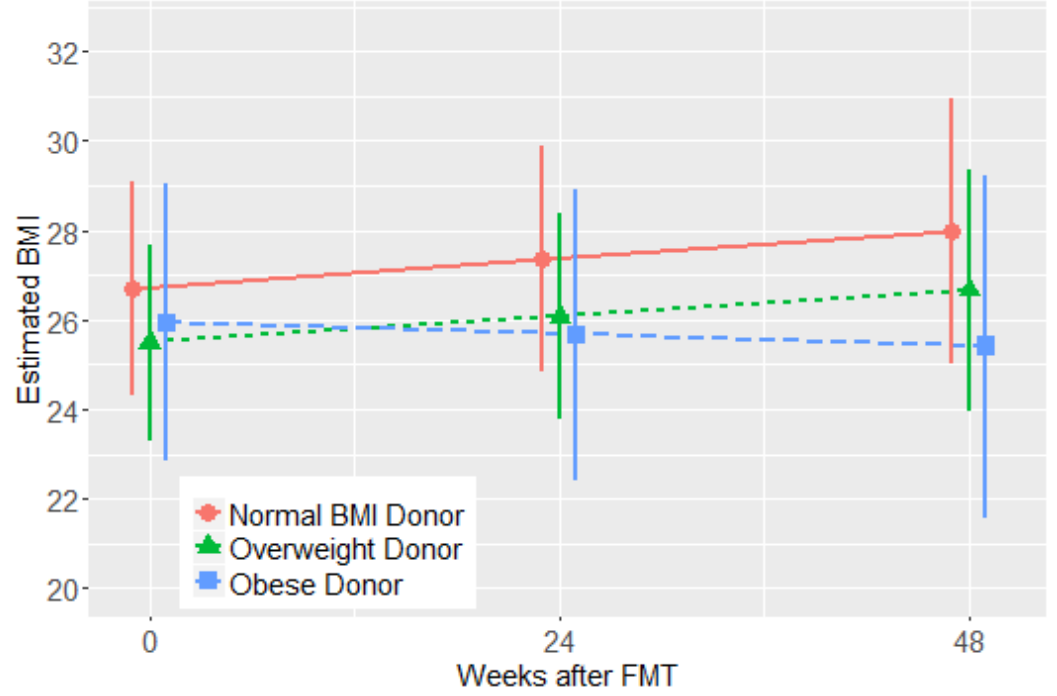

